\title{
Multi-echo, multi-slice, cardiovascular T2* spiral imaging in a single breath-hold
}

\author{
Nii O Addy*, R Reeve Ingle, Kenneth O Johnson, Galen D Reed, Michelle M Nystrom, William R Overall, \\ Juan M Santos, Bob S Hu \\ From 19th Annual SCMR Scientific Sessions \\ Los Angeles, CA, USA. 27-30 January 2016
}

\section{Background}

Elevated levels of iron in the body can be detected in various organs including the heart. If not treated, high iron levels can lead to serious health conditions including heart failure and cirrhosis. Cardiovascular MR provides a non-invasive and repeatable alternative method to endomyocardial biopsy for monitoring iron levels within the myocardium.

Multi-echo, single slice, Cartesian acquisitions have previously been developed for cardiovascular iron assessment with MR, but the presence of iron within the myocardium can be heterogeneous [1]. In this work, we present a single breath-hold, multi-echo, multi-slice, spiral acquisition providing large coverage of the heart for the assessment of cardiovascular iron deposition.

\section{Methods}

Imaging was performed on a $1.5 \mathrm{~T}$ GE Signa TwinSpeed scanner using the RTHawk Cardiac platform (HeartVista, Menlo Park, CA). A generalized pulse sequence diagram is shown in Figure 1 for $\mathrm{S}$ slices, $\mathrm{E}$ echoes and

a spiral trajectory with I interleaves. Data was acquired with an ECG-gated GRE sequence with parameters: $28 \times 28 \mathrm{~cm}^{2}$ FOV, $1.8 \times 1.8 \mathrm{~mm}^{2}$ spatial resolution, four TEs $(3.42 \mathrm{~ms}, 8.95 \mathrm{~ms}, 14.5 \mathrm{~ms}, 20.0 \mathrm{~ms}), 10 \mathrm{~mm}$ slice thickness, 4 spiral interleaves, $14.4 \mathrm{~ms}$ readout duration, and 1.2 acceleration factor. The desired number of slices to be acquired was determined by the user. Images were obtained within a single breath-hold by interleaving acquisitions from multiple slices within each heartbeat. Adjusting the number of slices affected the acquisition window within each heartbeat, but did not affect the overall scan time.

The sequence was tested on volunteers to demonstrate the feasibility of the technique. Images were reconstructed with partially parallel imaging with localized sensitivities.

\section{Results}

In volunteers 1 and 2, data was acquired in 16 and $20 \mathrm{~s}$ breath-holds, respectively. A single slice was imaged in volunteer 1 and 5 slices were imaged in volunteer 2 .

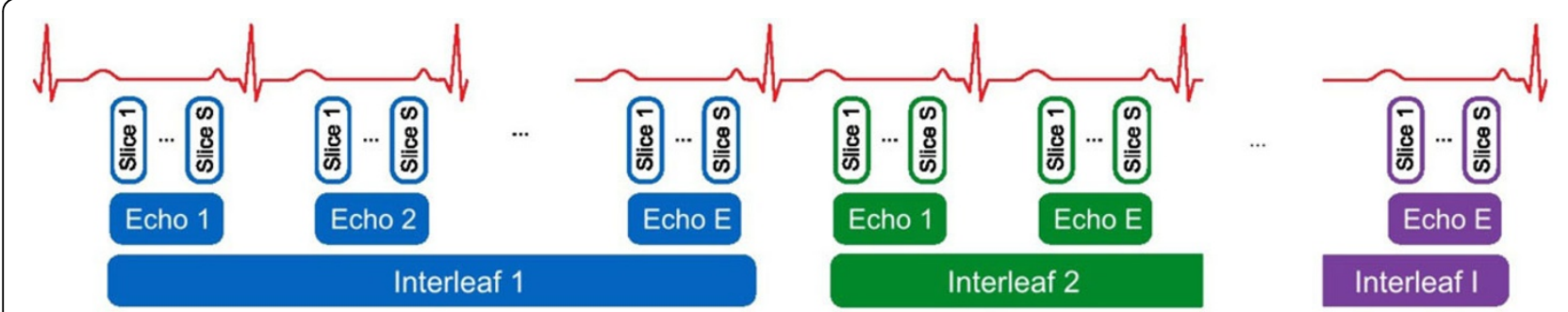

Figure 1 Pulse sequence diagram of the multi-echo, multi-slice imaging sequence acquiring $\mathrm{S}$ slices and $\mathrm{E}$ echoes using a spiral trajectory with I interleaves.

HeartVista, Inc, Menlo Park, CA, USA 


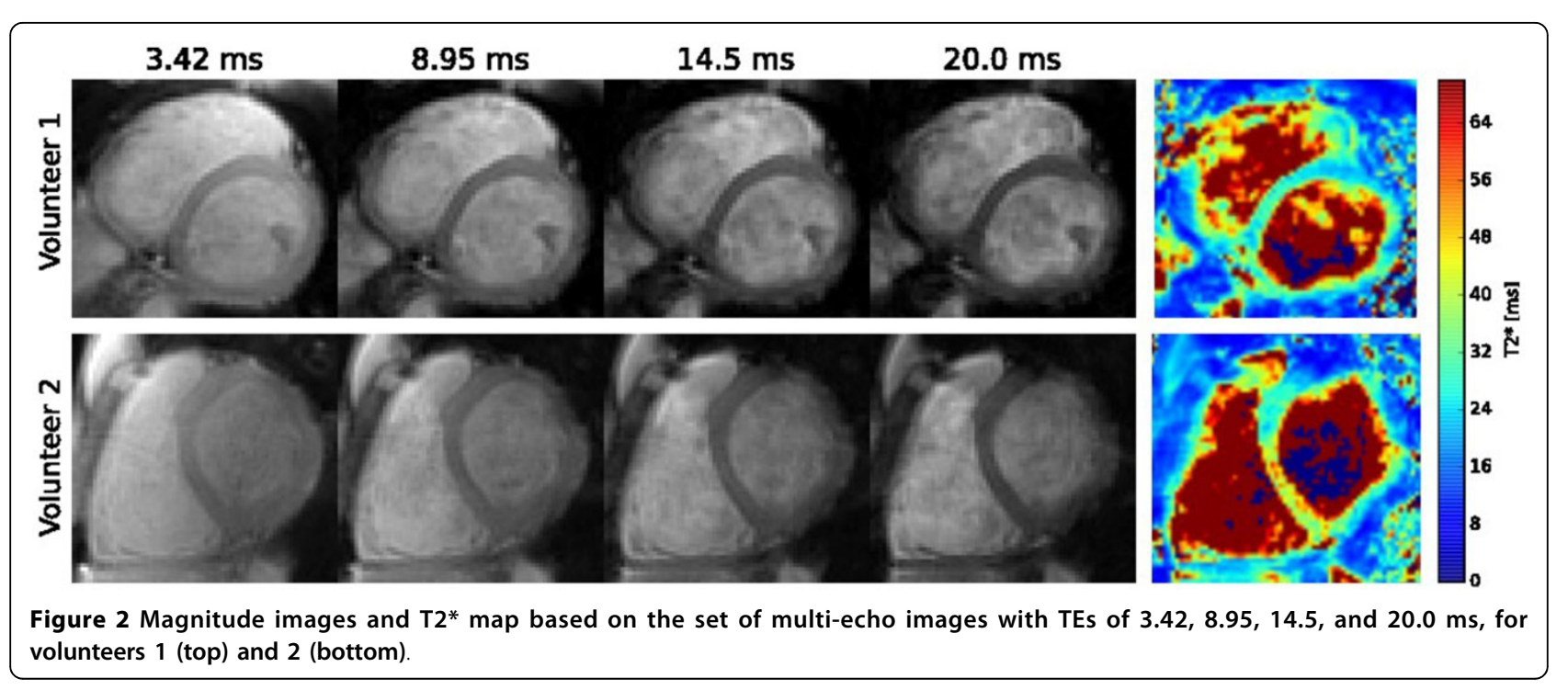

The images of the set of four TEs are shown in Figure 2. The signal intensity of the magnitude images were fit to a monoexponential curve $\mathrm{M}_{0} \mathrm{e}^{-\mathrm{TE} / \mathrm{T} 2^{*}}$ on a pixel-by-pixel basis to generate the $\mathrm{T} 2 *$ maps. $\mathrm{T} 2 *$ was measured in the interventricular septum, which is less prone to susceptibility artifacts and representative of $\mathrm{T} 2 *$ throughout the myocardium. The average $\mathrm{T} 2$ * values in the two volunteers were 25.3 and $36.2 \mathrm{~ms}$ with standard deviations of 3.3 and $8.5 \mathrm{~ms}$, respectively. Values greater than $20 \mathrm{~ms}$ are generally considered normal.

\section{Conclusions}

With spiral imaging, a set of $\mathrm{T} 2{ }^{*}$ weighted images can be acquired over multiple slices in a single breath-hold. Future work includes clinical evaluation. 